\title{
Paediatric Virology as a new educational initiative: An interview with Nobelist Professor of Virology Harald zur Hausen
}

\author{
IOANNIS N. MAMMAS and DEMETRIOS A. SPANDIDOS
}

Department of Clinical Virology, School of Medicine, University of Crete, Heraklion 71003, Greece

Received July 18, 2017; Accepted August 22, 2017

DOI: 10.3892/etm.2017.5006

\begin{abstract}
Born in Gelsenkirchen-Buer in Germany on March 11th, 1936, Professor Harald zur Hausen, Emeritus Professor of Virology at the University of Freiburg and 2008 Nobel Prize Laureate in Physiology or Medicine for his discovery of human papillomavirus (HPV), which causes cervical cancer, believes that good knowledge of virological methods and diagnostic possibilities are an asset for all young paediatricians. Professor zur Hausen considers that the creation of an educational platform on Paediatric Virology is definitely very beneficial for young paediatricians, as this will greatly enhance their knowledge in the field of Virology. He very actively advocates the vaccination of boys for the eradication of HPV infection and emphasises that male HPV vaccination should be included into the current vaccination programmes. He would have certainly considered Dr George N. Papanicolaou (Kyme, Island of Euboea, Greece, 1883 - Miami, Florida, USA, 1962) as an excellent candidate for the Nobel Prize, stating that the contribution of Dr Papanicolaou did not find sufficient recognition in the past. In the context of the 3rd Workshop on Paediatric Virology, which will be held in Athens, Greece, on October 7th, 2017, Professor zur Hausen will give his plenary lecture on 'Paediatric Virology and Oncology: Virus persistence and the important first years of life'.
\end{abstract}

\section{Contents}

1. Introduction

2. Questions and Answers

Correspondence to: Professor Demetrios A. Spandidos, Department of Clinical Virology, School of Medicine, University of Crete, Heraklion 71003, Greece

E-mail: spandidos@spandidos.gr

Key words: Paediatric Virology, Medical Education, human papillomavirus, Harald zur Hausen

\section{Introduction}

Born in Gelsenkirchen-Buer in Germany on March 11th, 1936, Professor Harald zur Hausen (Fig. 1) studied Medicine at the Universities of Bonn, Hamburg and Düsseldorf, and received his MD in 1960 (1-3). In 1966, he moved to the Children's Hospital of Philadelphia in the USA, where he joined the Laboratory of Dr Werner Henle and Dr Gertrude Henle and 2 years later he was appointed as an Assistant Professor of Virology at the University of Pennsylvania. In 1969, he returned to Germany, and after a period of 3 years as a senior scientist at the Institute of Virology of the University of Würzburg, he was appointed in 1972, at the age of 36, as a Chairman and Professor of Virology at the University of Erlangen-Nürnberg in Bavaria. In 1977, he moved to a similar position at the University of Freiburg and from 1983, until his retirement in 2003, he was appointed as a Scientific Director of the German Cancer Research Centre, Deutsches Krebsforschungszentrum (DKFZ) in Heidelberg. Nine years ago, on October 6th, 2008, Professor zur Hausen received the Nobel Prize in Physiology or Medicine for his discovery of human papillomavirus (HPV), which causes cervical cancer, a discovery that proved the principal role of HPV in cervical cancer and led to the development of the current vaccines against HPV.

His first experiments, which aimed at establishing an aetiological association between HPV and cervical cancer, were initiated in 1972. As his research group failed to find genetic DNA sequences for herpes simplex virus (HSV) type 2 in cervical cancer biopsies, he went against the dogma of that time, which considered that HSVs were associated with cervical carcinogenesis. This false dogma implicating HSVs had gained a catholic scientific acceptance since the end of the 1960s and had been supported by gynaecologists for more than 2 decades. His principal observation that HPV is not a single virus, but many with a major heterogeneity, led his research group to be the first, during the late 1970s, to isolate HPV 6 and HPV 11 from genital wart biopsies, and HPV 16 and HPV 18 from cervical cancer biopsies, and to hypothesise that cervical cancer is mainly caused by HPV. This hypothesis, which achieved a major breakthrough in the field of Virology and led to tremendous progress being made in women's health in the coming years, was completely novel and the scientific community at that time was not enthusiastic enough to accept the viral aetiology of cervical cancer. After the beginning of the vaccination period against HPV, HPV is well established 
as the virus of Professor zur Hausen, a great pioneer of modern Virology in the entire History of Medicine, who can be undoubtedly known as our 'Father of HPV Virology' (1).

To date, Professor zur Hausen, the Noblest of Nobel prize winners, has supported the Paediatric Virology Study Group (PVSG) since its birth and has offered his valuable expert advice to the Workshops on Paediatric Virology (4-7). In 2016, during the 2nd Workshop on Paediatric Virology, Professor zur Hausen was awarded the ' 2016 George N. Papanicolaou Humanitarian Award' for his outstanding academic, clinical, research and publishing contribution to the wealth, health and future of humanity (6), while in 2017, he will receive the '2017 Paediatric Virology Award in Virology' for his contribution on HPV research in childhood (8). In the context of the 3rd Workshop on Paediatric Virology, which will be held in Athens on October 7th, 2017, he will give his plenary lecture on 'Paediatric Virology and Oncology: Virus persistence and the important first years of life' and will focus on diet, cancer, neurodegenerative diseases and viruses (9).

\section{Questions and Answers}

Question: Professor Harald zur Hausen, first of all, thank you for your acceptance to co-chair the 3rd Workshop on Paediatric Virology entitled 'Paediatric Virology: Interaction between basic science and clinical practice', which will be held in Athens on October 7th, 2017. In our previous meeting in Heidelberg in 2014, you considered that viral infectious diseases play a significant role in Paediatrics and you were very encouraging of our will at that time to create an educational platform on Paediatric Virology. One year after your advice, the Workshop on Paediatric Virology was created, which was held in Athens in 2015, for the first time, and in 2016. Based on the edition supplements of our 1st and 2nd Workshops on Paediatric Virology, we would like to have your feedback and comments on these.

Answer: Indeed, up to today, I believe that the creation of an educational platform on Paediatric Virology would be very beneficial. The material, which is sent to me at this stage, is a very good indication for the role of this field in Paediatrics.

Question: During the past two decades, the new advances on the field of Virology have expanded the level of knowledge on viral infections affecting neonates and children. Do you consider educationally important for young paediatricians to have good knowledge on Virology?

Answer: Yes, I believe that it is particularly important for young paediatricians to have a good knowledge of the field of Virology.

Question: Your statement two years ago was that Paediatric Virology should not be separated as a subspecialty from Paediatric Infectious Diseases. Which role do you believe that Paediatric Virology could play in the Paediatric Infectious Diseases section?

Answer: I still think that Paediatric Virology should not be separated from Paediatric Infectious Diseases. I believe, however, that good knowledge of virological methods and diagnostic possibilities would be an asset for all young paediatricians.

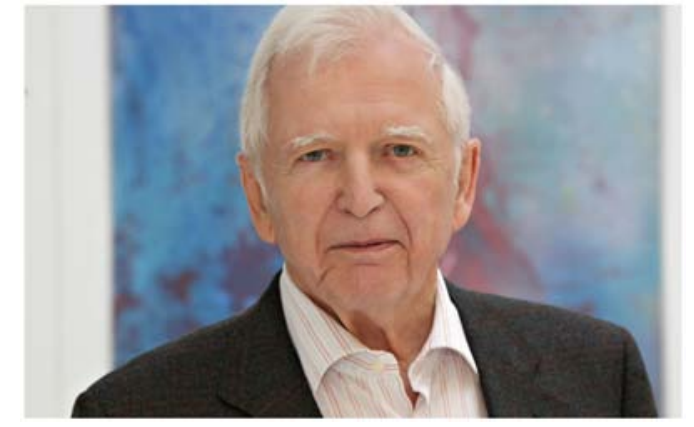

Figure 1. Professor Harald zur Hausen, 2008 Nobel Laureate, Emeritus Professor of Virology at the University of Freiburg, Germany and Chair of the 3rd Workshop on Paediatric Virology, October 7th, 2017, Athens, Greece.

Question: How do you explain the presence of oncogenic HPV DNA in neonates, a finding, which has been published in several studies during the past 10 years?

Answer: There exists the possibility that newborn babies are infected by passing through the birth channel. It seems, however, from the studies available to me, that the risk of maintaining this infection is very low for this age group. They probably lose it during their first year of life. This may be in part due to the fact that high-risk HPVs are to a certain extent hormone-responsive, which could be neatly demonstrated by in vitro studies.

Question: Is it time to include HPV vaccination during the first year of life?

Answer: I don't believe that it be worth to include babies in HPV vaccination during the first year of life for the reasons outlined in the previous point.

Question: We would like to have your comment on the necessity of including boys into the current vaccination programmes against HPV.

Answer: Yes, I very actively advocate the vaccination of boys as well. It is quite clear that males between 15-40 years of age have more sexual partners than females of the same age group. If you really wish to eradicate these viral infections, you need to include boys into the vaccination programmes. Moreover, due to the fact that oropharyngeal carcinomas and anal carcinomas are also, to a large degree, caused by HPV 16 and 18 infections, we need to stress the vaccination of boys. The age group should be the same (9-14 years of age).

Question: Your point about male vaccination against HPV is essential and it will be discussed at the workshop on October; however, what should be the optimal medical practice in Greece, a country under continuous financial crisis?

Answer: I strongly recommend negotiations of your Health Ministry or of your health insurances with the respective companies. Many countries in a rather critical financial situation manage to receive a rather substantial reduction of the prices for the vaccine.

Question: What is the potential role of the new oncogenic viruses that are under your investigation during the last years in childhood?

Answer: I will cover this in my talk. I am still reluctant to consider them as 'new oncogenic viruses'. In our studies evidence is pretty good for multiple sclerosis. 
Question: As the '2016 George N. Papanicolaou Humanitarian Award' recipient, we would like to have your comment on the contribution of Dr George N. Papanicolaou in Medicine. Answer: I stated on many public occasions that the contribution of Dr George N. Papanicolaou did not find sufficient recognition in the past. Indeed, he contributed substantially to the decline of cervical cancer in many of those countries, which followed his steps and had a careful analysis of cervical smears.

Question: Do you believe that Dr George N. Papanicolaou should have received the Nobel Prize? Which do you believe that the main reasons were for not receiving this prize? If he was contemporary with you, would you have proposed him for this prize?

Answer: He clearly would have been an excellent candidate for the Nobel Prize. I am not fully able to define the main reasons for not receiving this prize. One thing, which is highly apparent to me, is that many of the European pathologists and cytologists were at that stage not convinced that this message would really provide a basis to reduce the cancer load. I certainly would have considered him for this prize.

Question: Thank you indeed for your answers and your continuous support; we are looking forward to your chairship and your plenary lecture on October in Athens.

\section{References}

1. Mammas IN and Spandidos DA: Four historic legends in human papillomaviruses research. J BUON 20: 658-661, 2015.

2. zur Hausen H: Papillomaviruses and cancer: From basic studies to clinical application. Nat Rev Cancer 2: 342-350, 2002.

3. zur Hausen H: HPV vaccines: What remains to be done? Interview by Lauren Constable. Expert Rev Vaccines 10: 1505-1507, 2011.

4. Greenough A, Theodoridou M, Kramvis A, Mammas IN, Christaki I, Koutsaftiki C, et al: Workshop on Paediatric Virology. Paediatric Virology Study Group, Athens, October 10, 2015.

5. Mammas IN, Greenough A, Theodoridou M, Kramvis A, Christaki I, Koutsaftiki C, Koutsaki M, Portaliou DM, Kostagianni G, Panagopoulou P, et al: Current views and advances on Paediatric Virology: An update for paediatric trainees. Exp Ther Med 11: 6-14, 2016.

6. Thiagarajan P, Gardner S, Theodoridou M, Kramvis A, Melidou A, Papaioannou G, et al: 2nd Workshop on Paediatric Virology. Paediatric Virology Study Group, Athens, October 8, 2016.

7. Mammas IN, Theodoridou M, Kramvis A, Thiagarajan P, Gardner S, Papaioannou G, Melidou A, Koutsaki M, Kostagianni G, Achtsidis V, et al: Paediatric Virology: A rapidly increasing educational challenge. Exp Ther Med 13: 364-377, 2017.

8. Pfister $\mathrm{H}$ and zur Hausen H: Seroepidemiological studies of human papilloma virus (HPV-1) infections. Int J Cancer 21: 161-165, 1978.

9. Mammas IN and Spandidos DA: Athens-based meeting to discuss the paediatric virology crossroad in October 2017. Acta Paediatr 106: 1536, 2017. 